\title{
Brightness as a function of retinal locus'
}

LAWRENCE E. MARKS ${ }^{2}$

LABORATORY OF PSYCHOPHYSICS, HARVARD UNIVERSITY

Brightness functions were determined for the dark-adapted fovea and periphery. In one series of experiments, observers matched numbers to the brightness of a $1^{\circ}$ white target at various intensities, presented half the time to the fovea, the other half to one of five peripheral loci: $5^{\circ}, 12^{\circ}, 20^{\circ}, 35^{\circ}$, and $6 n^{\circ}$. In a second series, observers matched the brightness of a $1^{\circ}$ white target in the fovea of one eye to the brightness of an identical target in the periphery of the other eye at various intensities. Thresholds were also determined for the fovea and for the five peripheral loci by a staircase procedure. The magnitude estimations and the interocular matches concur in showing that a stimulus of fixed luminance appears brighter in the periphery than in the fovea. The brightness was found to be maximal at $20^{\circ}$. Brightness grows as a similar power function of luminance at all six retinal positions.

Several studies have measured the absolute threshold as a function of retinal locus (see, for example, Wentworth, 1930; Hecht, Haig, \& Wald, 1935; Crozier \& Holway, 1939), but few, if any, have been concerned with the brightness of supraliminal stimuli at different retinal loci. Troland (1930) wrote that "the brilliance which is evoked by a stimulus of fixed photometric intensity varies with position in the field of vision, being, in general, greater in the periphery than in the center" (p. 81). Troland regretted that, though his statement concerned supraliminal brightness, the only facts he could cite were measurements of absolute thresholds. The present study was an attempt to measure the brightness functions for supraliminal stimuli at a number of retinal loci. The methods used were magnitude estimation, in which observers matched numbers to the brightness of peripheral and foveal targets, and interocular matching, in which observers matched the brightness of peripheral to foveal and of foveal to peripheral targets.

Measurements of absolute thresholds show that, for the dark-adapted eye, the peripheral threshold for white light is lower than the foveal threshold; furthermore, the threshold passes through a minimum as the target is moved away from the fovea (Stiles \& Crawford, 1937; Zigler \& Wolf, 1958; Wolf \& Zigler, 1959). Stiles and Crawford found the minimum to be as near to the fovea as $8^{\circ}$ and as distant as $20^{\circ}$ for different observers. In the two studies by Wolf and Zigler, the minimum was found to occur at about $10^{\circ}$ from the fovea.

For colored lights the situation is more complex. Wentworth (1930) found that the most sensitive region of the retina for narrow spectral bands in the red, yellow, green, and blue ranges occurred at loci of $25^{\circ}$ to $40^{\circ}$ from the fovea. Pirenne (1948), on the other hand, found that sensitivity to the red region was greatest in the fovea, and that the threshold decreased steadily with increased eccentricity of locus. For light in the blue region, however, he noted that the threshold was lowest at a point about $20^{\circ}$ from the fovea. In fact, Hecht, Schlaer, and Pirenne (1941), in their attempt to determine the minimal energy necessary for a threshold response under optimal conditions, used light of $510 \mathrm{~nm}$ placed $20^{\circ}$ from the fovea.

It is striking that the differences between foveal and peripheral threshold levels depend markedly upon stimulus size. For fields subtending moderate visual angles $\left(2^{\circ}\right.$ to $\left.3^{\circ}\right)$, the difference between the foveal and the minimum peripheral thresholds is about 20 to $30 \mathrm{~dB}$ (Zigler \& Wolf, 1958; Hecht, Haig, \& Wald, 1935; Troland, 1930), whereas small fields $\left(4^{\prime}\right.$ to $\left.20^{\prime}\right)$ tend to produce a difference of only about $10 \mathrm{~dB}$ (Wolf \& Zigler, 1959; Stiles \& Crawford, 1937; Crozier \& Holway, 1939). In one experiment, in which the visual angle of the target subtended $2.7 '$, no difference at all was found between foveal and peripheral thresholds at a locus $8^{\circ}$ from the fovea (Arden \& Weale, 1954). There is abundant evidence that foveal brightness grows as a power function of luminance. For the darkadapted eye and for targets larger than about $1^{0}$, the exponent of the power function is about 0.33 (Stevens \& Stevens, 1963; Onley, 1964). The psychophysical power function, which appears to be a general law applicable to all prothetic continua, can be expressed as

$$
\psi=k\left(\phi-\phi_{0}\right) \beta,
$$

where $\psi$ is brightness, $\phi$ is luminance, $\phi_{0}$ is a constant that seems to depend upon the threshold, $\mathrm{k}$ is a constant that depends upon the choice of units, and $B$ is the exponent (Stevens, 1961).

If the growth of brightness follows a power function at any retinal locus, then three parameters of the brightness equation, $\mathbf{k}, \phi_{0}$, and $\beta$, may be compared for the various loci. It is to be expected, of course, that $\phi_{o}$ will vary with retinal locus. If this were the only variation, the brightness functions for peripheral targets should appear almost identical to one another and to the foveal function over most of the intensity range, because the threshold constant becomes negligible at stimulus levels 10 to $15 \mathrm{~dB}$ above the threshold. If $\mathbf{k}$ varies, the apparent brightnesses produced by equal stimuli at two different loci would bear a constant ratio to each other. If the exponent $\beta$ varies with locus, the power functions would have different slopes in a log-log plot.

Two sets of experiments were conducted in order to compare the peripheral and foveal brightness functions. One set consisted of five magnitude estimation experi- 


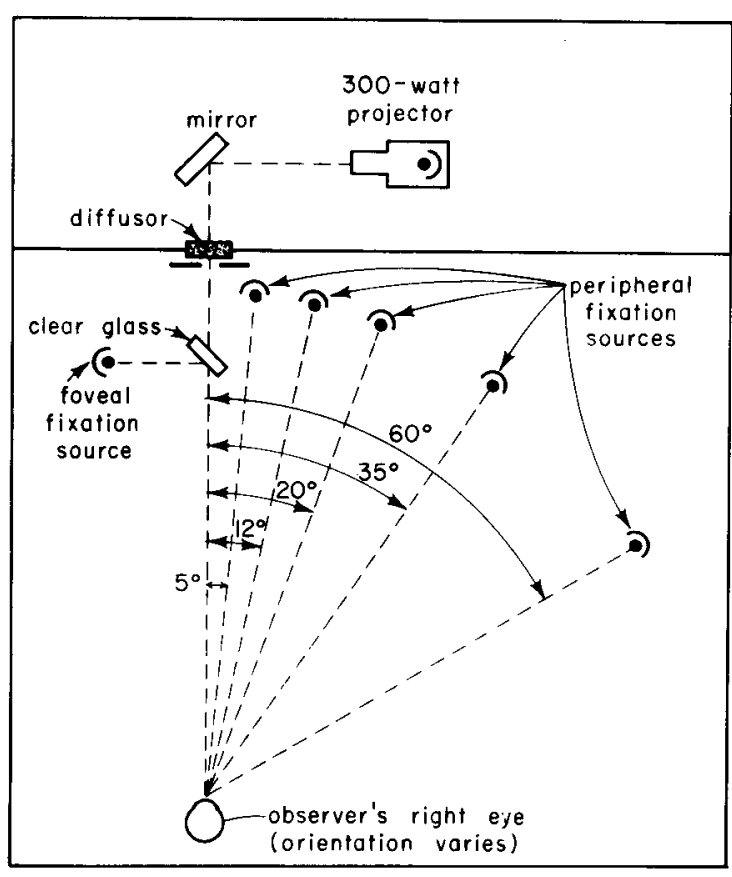

Fig. 1. The principal parts of the apparatus for the magnitude estimation and threshold experiments (not to scale).

ments. In each experiment observers were presented targets of white light at a number of luminance levels; half the presentations were viewed foveally, half at a particular peripheral locus. The observers were asked to assign numbers proportional to the apparent brightness of the stimuli. In the second set of five experiments, observers matched the brightness of peripheral to foveal targets and foveal to peripheral targets. The peripheral loci examined were the same five used in the magnitude estimation experiments. In addition, absolute thresholds were measured at these loci.

\section{EXPERIMENT I: MAGNITUDE ESTIMATIONS Apparatus}

The apparatus is diagrammed in Fig. 1. The white light from a 300-watt bulb was reflected through an aperture into a light-proof room. A disc of milkPlexiglas in the path of the beam diffused the light. The aperture was $0.75 \mathrm{~cm}$ in diameter, and the visual angle subtended by the target was $1^{\circ}$ when the observer's eye was $43 \mathrm{~cm}$ from the aperture. The luminance of the unattenuated light was $105 \mathrm{~dB}$ re $10^{-10}$ lambert. This luminance level could be reduced by means of neutral density filters inserted in the path of the target.

A small red light appeared near the center of the target and served as the foveal fixation point. This light was produced by a small bulb located to the right of the target and reflected by a thin piece of clear glass. Other bulbs were arranged in the chamber such that, when one of them was viewed by the observer, the target light would appear in the periphery. The optical distance from the observer's eye to each fixation light was $43 \mathrm{~cm}$.

\section{Procedure}

After dark-adapting for $10 \mathrm{~min}$. by wearing red goggles, the observer fixed his head in a chin-rest, to which was attached a piece of black cardboard that prevented the left eye from seeing the fixation and target lights. Six levels of luminance were used, from 55 to $105 \mathrm{~dB}$ re $10^{-10}$ lambert in 10-dB steps. Each level was presented four times, twice viewed foveally, twice peripherally, in irregular order. When the presentation was to be foveal, the fixation light that appeared directly in the path of the target was presented; when the presentation was to be peripheral, the appropriate peripheral fixation point appeared to the right of the target. Since the fixation points and target were viewed with the right eye only, the peripheral targets all fell on the temporal portion of the horizontal meridian of the retina.

In a given session, only the fovea and one peripheral locus were stimulated. The five peripheral loci were $5^{\circ}, 12^{\circ}, 20^{\circ}, 35^{\circ}$, and $60^{\circ}$. In each session, the observer was first given a stimulus of moderate intensity and told to assign to it a number that seemed appropriate to its subjective brightness; then, for each subsequent stimulus, he was to assign a number whose proportion to the first number reflected the ratio of the subjective brightnesses. The duration of each stimulus presentation was 0.5 sec., and intervals of about $20 \mathrm{sec}$. separated presentations. Twelve observers took part in each session, drawn randomly from a total of 22. All had previous experience in making magnitude estima-

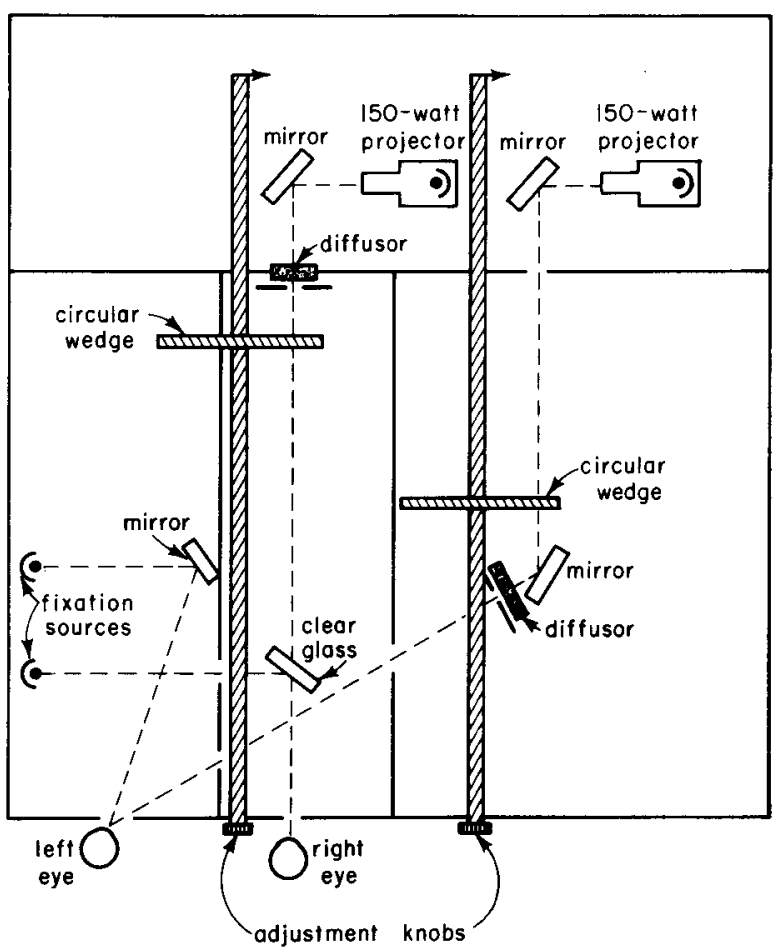

Fig. 2. The principal parts of the apparatus for the brightness matching experiment (not to scale). 


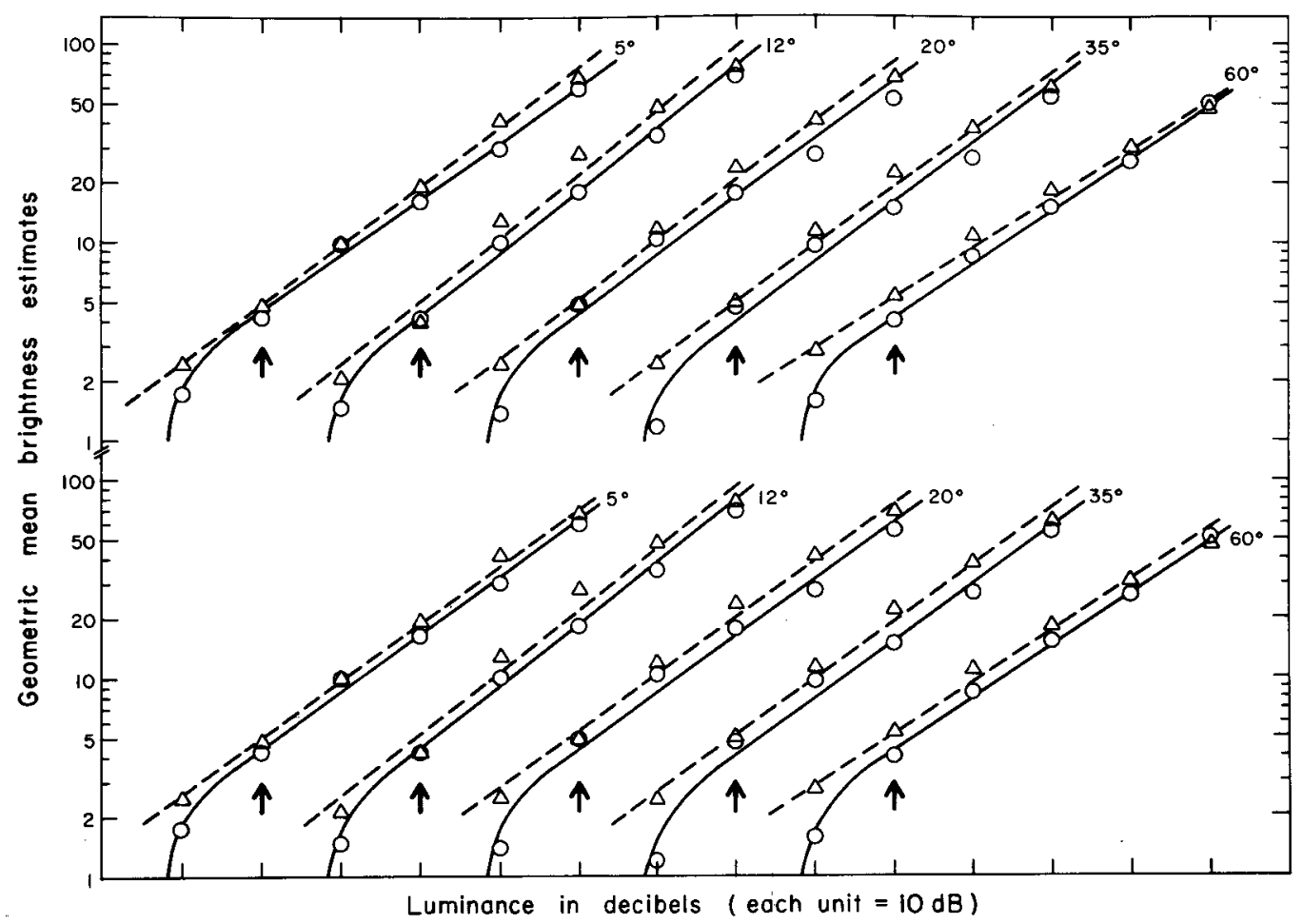

Fig. 3. Geometric means of the magnitude estimates of brightness for peripheral targets (dashed lines) and foveal targets (solid lines). Each point is the mean of 24 judgments. The upper sets of points were fitted by the method of least squares, with a value equal to $53 \mathrm{~dB}$ subtracted from all the foveal luminances. The lower sets of lines were fitted so that the two functions in each pair have the same slope. The arrows indicate luminances of 65 dB re $10^{-10}$ lambert.

tions. In each session the first stimulus presented was foveal for half the observers, peripheral for the other half.

\section{EXPERIMENT II: BRIGHTNESS MATCHES \\ Apparatus}

Figure 2 is a diagram of the apparatus. The white light from a 150-watt bulb was reflected through an aperture into a dark booth. A disc of milk-Plexiglas, placed at the aperture, served to diffuse the light. The aperture was $0.6 \mathrm{~cm}$ in diameter, and the visual angle subtended by the target was $1^{\circ}$ when the observer's eye was $34 \mathrm{~cm}$ from the aperture. A neutraldensity optical wedge was mounted between the observer's eye and the target. This permitted a continuous attenuation of the luminance over a range of about $40 \mathrm{~dB}$. Additional attenuation was possible by means of neutral-density filters. A similar arrangement produced a second target. The position of this second target could be varied but was always to the right of the first target, as viewed by the observer. The target at the left served as the foveal target, which was viewed by the right eye, the target at the right as the peripheral target, which was viewed by the left eye. The angle of separation between the foveal and peripheral targets was controlled by a mirror. By varying the positions of the diffusor and aperture, it was possible to keep a constant optical distance of $34 \mathrm{~cm}$ from the observer's left eye to the target and a visual angle of $1^{\circ}$ for the target.

Fixation lights, at an optical distance of $34 \mathrm{~cm}$, appeared at the center of the left (foveal) target. A piece of clear glass, inserted in the path of the foveal target, reflected the light from a small red bulb set off to the side. This fixation point was viewed by the right eye only. The light from another small bulb was reflected by a mirror to the left eye only. The observer was required to fuse the two fixation lights, i.e., the two eyes were converged as if the foveal target were being viewed by both eyes. This helped to stabilize the left eye.

\section{Procedure}

The observer was first dark-adapted for $10 \mathrm{~min}$. by wearing red goggles, then was seated in the dark booth, his head in a chin-rest and his right eye $34 \mathrm{~cm}$ from the foveal target. Timers connected to the projectors turned on the two target lights simultaneously; the targets were on for $0.75 \mathrm{sec}$. and off for $9.25 \mathrm{sec}$. in a repeated cycle. In any one session, the fovea and one peripheral locus were stimulated; the five peripheral loci were again $5^{\circ}, 12^{\circ}, 20^{\circ}, 35^{\circ}$, and $60^{\circ}$ from the fovea in the temporal portion of the horizontal meridian of the retina.

Six observers served in all five sessions. Only two had served previously in psychophysical experiments. In one part of a session the observer adjusted the 
Table 1. Exponents of the Foveal and Peripheral Brightness Functions, from Magnitude Estimation Experiments

\begin{tabular}{ccc} 
Peripheral target locus & Foveal exponent & Peripheral exponent \\
\hline $5^{\circ}$ & 0.28 & 0.30 \\
$12^{\circ}$ & 0.31 & 0.32 \\
$20^{\circ}$ & 0.28 & 0.29 \\
$35^{\circ}$ & 0.29 & 0.28 \\
$60^{\circ}$ & 0.27 & 0.25 \\
\hline
\end{tabular}

foveal to match the peripheral target, and in the other part he adjusted the peripheral to match the foveal target. Five luminance levels between 55 and $95 \mathrm{~dB}$ re $10^{-10}$ lambert, separated by $10-\mathrm{dB}$ steps, were presented in irregular order in any one part of a session. About $25 \mathrm{sec}$. elapsed between finishing one brightness match and starting the next. For each peripheral locus three observers first matched foveal to peripheral brightness; the other three first matched peripheral to foveal. In each part of each session, three matches were made to each of the five fixed luminances presented.

\section{ABSOLUTE THRESHOLDS}

Absolute thresholds were measured at the fovea and $5^{\circ}, 12^{\circ}, 20^{\circ}, 35^{\circ}$, and $60^{\circ}$ from the fovea for two of the observers who served in Experiment II. The apparatus, fixation points, and position of the observer were the same as those used in Experiment I (Fig. 1), except that a neutral-density optical wedge was added.

The observer was first dark-adapted for $10 \mathrm{~min}$. with red goggles. Thresholds were determined by a staircase procedure. The first stimulus presented was well above the absolute threshold; if the observer reported that he saw it, the intensity was reduced by $2.5 \mathrm{~dB}$ and the stimulus was presented again. This continued until the observer reported that he did not
Table 2. Number of Decibels Separating Foveal and Peripheral Brightness Functions. Data from magnitude estimation experiments

\begin{tabular}{cc} 
Peripheral target locus & Decibel separation \\
\hline $5^{\circ}$ & 1.8 \\
$12^{\circ}$ & 2.1 \\
$20^{\circ}$ & 3.4 \\
$35^{\circ}$ & 3.3 \\
$60^{\circ}$ & 3.1 \\
\hline
\end{tabular}

see the target; then the intensity was increased by $2.5 \mathrm{~dB}$ for the next presentation. This was continued until 15 reversals of intensity had occurred. Then the procedure was repeated in the same session for a different retinal position until all six retinal positions were finished. The duration of each stimulus presentation was $0.75 \mathrm{sec}$.

\section{Magnitude Estimations}

\section{RESULTS}

The geometric means of the brightness estimates for the peripheral and foveal stimuli for each peripheral position are plotted in the upper portion of Fig. 3. Each set of points seems to be described fairly well by a power function (a straight line in log-log coordinates). The lower ends of the foveal functions show the curvature frequently seen when the threshold constant $\left(\phi_{0}\right)$ is not subtracted from the physical intensity. If luminance were measured from the threshold, the functions would approximate straight lines throughout their entire length.

The slopes (exponents of the power functions) were calculated for all five foveal functions by the method of least squares, using various values for $\phi_{0}$ between 50 and $54 \mathrm{~dB}$. Since the value of $53 \mathrm{~dB}$ resulted in the smallest average sum of squared residuals for the five foveal functions, the least-squares solutions using

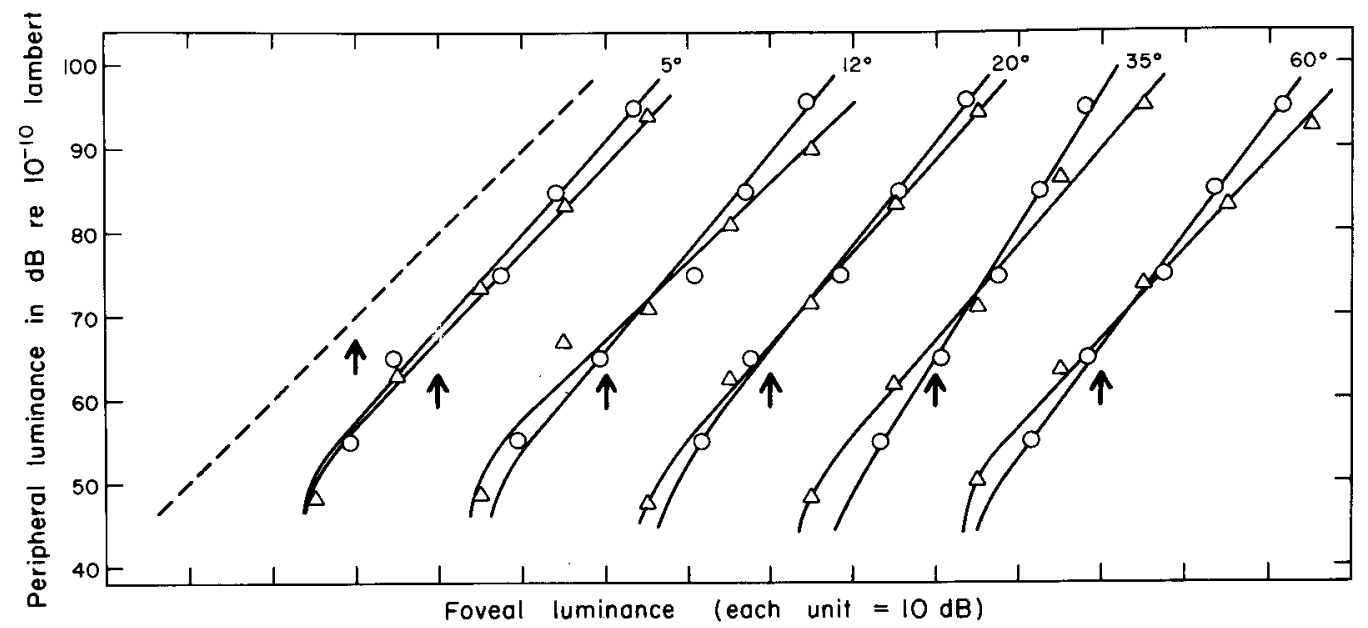

Fig. 4. Mean decibel settings for the adjustment of foveal to peripheral brightness (circles) and peripheral to foveal brightness (triangles). Each point is the mean of 18 settings. The peripheral loci are as indicated. The dashed line has unit slope and indicates equal foveal and peripheral luminances. The functions were fitted by the method of least squares with a value equal to $53 \mathrm{~dB}$ subtracted from all of the foveal luminances. The arrows indicate foveal luminances of $70 \mathrm{~dB}$ re $10^{-10}$ lambert. 
that value are plotted in the upper portion of Fig. 3, along with the least-squares solutions for the five peripheral functions. (N.B. the subtraction of $\phi_{0}$ is in linear units.) For each of the pairs of functions the value of the peripheral exponent is practically identical to the value of the foveal exponent (Table 1). In order to visualize more clearly the differences between the intercepts for the foveal and peripheral functions, the geometric means of the brightness estimates are plotted again in the lower part of Fig. 3 and fitted by the bestfitting lines of the same slope for each pair of functions. All of the peripheral functions (dashed lines) are displaced upward from the corresponding foveal functions, which shows that light seen by the periphery appears brighter than the same light seen by the fovea. Table 2 shows the number of decibels that separate each foveal from the corresponding peripheral function. The separation increases as the peripheral locus is moved out to $20^{\circ}$ from the fovea, then decreases as the peripheral locus is moved farther out.

\section{Brightness Matches}

The decibel averages of the matching luminances are plotted in Fig.4. As the power law predicts, straight lines in log-log coordinates fit the data fairly well, except for the lower ends of the functions where the effect of the threshold constant $\left(\phi_{0}\right)$ appears. The functions were fitted by the method of least squares, using $53 \mathrm{~dB}$ as the value to determine the $\phi_{0}$ to be subtracted from the foveal luminances. The dashed line in the figure shows an example of equal foveal and peripheral luminances. The entire lengths of nearly all the matching functions fall below the appropriate line of equal foveal and peripheral luminances, implying again that stimuli of equal brightness appear subjectively brighter in the periphery than in the fovea. In other words, for equal brightness the stimulus to the fovea must be more intense.

The pairs of functions in Fig. 4 show the ubiquitous "regression" phenomenon; that is to say, when the foveal target is adjusted, the functions are steeper and when the peripheral target is adjusted, the functions are flatter. This is another example of the general tendency, encountered in numerous psychophysical procedures, for observers to shorten the range of whichever stimulus is under their control (Stevens, 1956, 1959). A slope of unity for the overall matching

Table 3. Ratios of Foveal to Peripheral Exponents from Matching Experiments

\begin{tabular}{cccc}
$\begin{array}{c}\text { Peripheral } \\
\text { target locus }\end{array}$ & Foveal adjusted & Peripheral adjusted & Average \\
\hline $5^{\circ}$ & 1.11 & 1.05 & 1.08 \\
$12^{\circ}$ & 1.12 & 0.92 & 1.02 \\
$20^{\circ}$ & 1.19 & 1.07 & 1.13 \\
$35^{\circ}$ & 1.59 & 1.11 & 1.35 \\
$60^{\circ}$ & 1.32 & 1.00 & 1.16 \\
\hline
\end{tabular}

Table 4. Measured Absolute Thresholds in $\mathrm{dB}$ re $10^{-10}$ Lambert

\begin{tabular}{lcccccc} 
Observer & \multicolumn{6}{c}{ Retinal locus } \\
\hline & Fovea & $5^{\circ}$ & $12^{\circ}$ & $20^{\circ}$ & $35^{\circ}$ & $60^{\circ}$ \\
\hline MK & 49 & 35 & 31 & 24 & 35 & 48 \\
BB & 50 & 31 & 30 & 23 & 30 & 45
\end{tabular}

functions (average of the foveal-adjusted and peripheral-adjusted) would imply that the slopes of the peripheral and foveal functions are the same. There is a slight asymmetry, however, as shown by the averages in Table 3.

\section{Absolute Thresholds}

Absolute thresholds (geometric averages of the last 10 intensity reversals for each retinal locus) are given in Table 4. Clearly the threshold was lowest when the stimulus appeared $20^{\circ}$ from the fovea, rising again for positions farther away, until the foveal level itself was almost reached at $60^{\circ}$ from the fovea.

\section{DISCUSSION}

The results of the magnitude estimation and brightness matching concur in showing that a stimulus of given luminance appears brighter in the periphery than in the fovea. Furthermore, the evidence from both experiments suggests that brightness grows as a power function of luminance at all retinal loci. It is not clear, however, that the exponents of all of the power functions are exactly the same. The results from the magnitude estimations show no difference between the foveal exponent and the peripheral exponents, but the brightness matches suggest that the peripheral exponents may be somewhat smaller than the foveal exponent. This difference may be due to an asymmetry between the two parts of the matching experiment, the matching of foveal to peripheral and of peripheral to foveal brightness. Since brightness discriminability is poorer in the periphery than in the fovea (Stiles \& Crawford, 1934), the matching of peripheral to foveal brightness may not have shown the regression effect as in the reverse procedure. From Table 3 it can be seen that the exponents of the matching functions for the adjustment of peripheral to foveal brightness differ little from unity.

On the assumption that the matching functions have exponents of unity, a calculation was made of the number of decibels that separate the best-fitting function of unit. slope for each pair of matching functions and a line of equal foveal and peripheral luminances, where the effect of the foveal $\phi_{0}$ is negligible (Table 5). This is equivalent to the average difference between foveal and peripheral luminance when the two targets are of equal brightness. As with the functions derived from magnitude estimations, the decibel separation can be seen to increase as the eccentricity of 
Table 5.

Number of Decibels between Foveal and Peripheral Luminances at Equal Brightness, from the Averages of the Brightness Matches

\begin{tabular}{cc} 
Peripheral torget locus & Decibel difference \\
\hline $5^{\circ}$ & 1.2 \\
$12^{\circ}$ & 2.8 \\
$20^{\circ}$ & 2.4 \\
$35^{\circ}$ & 1.5 \\
$60^{\circ}$ & 1.4 \\
\hline
\end{tabular}

the peripheral stimulus is increased, and then to decrease again with further increase in eccentricity. The position of maximum decibel separation was $12^{\circ}$ in the matching experiments (Table 5), whereas the maximum occurred at $20^{\circ}$ in the magnitude estimation experiments (Table 2).

Another way to look at the averages of the two experiments is to construct the family of equalbrightness contours (Fig. 5). The upper four curves show the averages of the decibel separations determined from the interocular matches and magnitude estimates. It is clear that, as the stimulus moves away from the fovea, the luminance necessary to keep a constant brightness becomes less than at the fovea. For all the curves, $20^{\circ}$ marks the position for the minimum luminance; at greater eccentricities the luminance necessary for constant brightness levels increases.

In the lower portion of Fig. 5 are plotted the average thresholds measured in this experiment, plus threshold

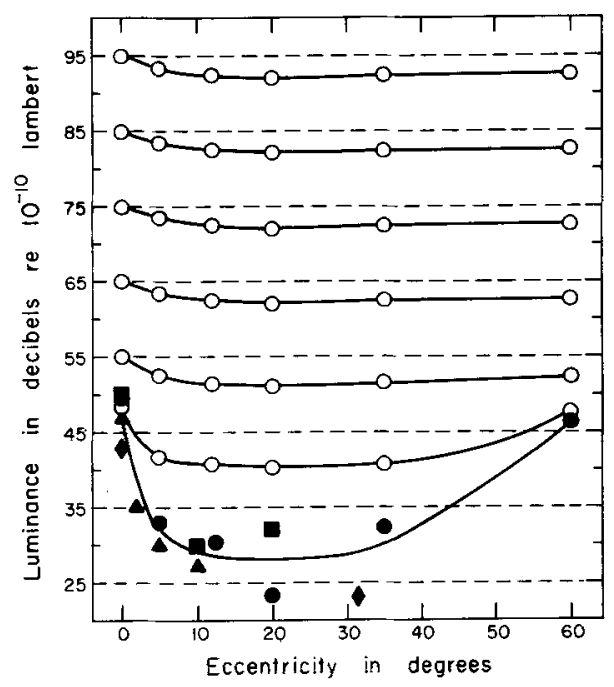

Fig. 5. Equal-brightness contours for targets viewed at various retinal loci. The four upper curves are based upon the averages of the magnitude estimation and brightness matching experiments. The filled symbols are threshold measurements: the circles are the averages of the thresholds measured in this study, the triangles are from Hecht, Haig, and Wald (1935), the diamonds from Graham, Brown, and Mote (1939), and the squares from Zigler and Wolf (1958). The measurements from Zigler and Wolf have all been lowered by $19 \mathrm{~dB}$ (see text). The two curves immediately above the threshold curve are based on interpolations. measurements made by Hecht, Haig, and Wald (1935), Graham, Brown, and Mote (1939), and Zigler and Wolf (1958). The measurements by Zigler and Wolf have all been lowered by $10 \mathrm{~dB}$, since their stimulus presentation was of brief $(40 \mathrm{msec}$ ) duration. The threshold curve also shows a minimum luminance near $20^{\circ}$. The two curves directly above the threshold curve were determined by interpolation with the aid of the supraliminal brightness functions and the threshold values. Thus, these two curves are similar in shape to the others, but the decibel differences are intermediate between the differences at threshold and the differences at high brightness levels. Table 6 gives the constants of the foveal and peripheral brightness functions that correspond to the equal-brightness contours of Fig. 5, assuming an exponent of 0.33 for all the functions.

A striking feature of Fig. 5 is the difference between the threshold contour and the equal-brightness contours. The threshold at the fovea is about $20 \mathrm{~dB}$ higher than the threshold at $20^{\circ}$; yet the luminance difference between the fovea and $20^{\circ}$ for equal brightness at high brightness levels is only about $3 \mathrm{~dB}$. Clearly, the relative sensitivity of various portions of the retina is greatly dependent upon brightness level. The absolute threshold contour is only one of an infinitude of equal-brightness contours that can be constructed and is thus only a single measure of the relative sensitivity of the retina. At brightness levels well above the absolute threshold, the differences in sensitivity across the retina are severely diminished.

A final point to be noted is that the foveal and peripheral brightness functions were all measured with the dark-adapted eye. Even the main finding that the same stimulus appears brighter in the periphery than in the fovea may not hold for the light-adapted eye. Troland (1930) believed that a stimuius of fixed luminance may appear brighter in the fovea of the light-adapted eye. Although there is no evidence with regard to supraliminal brightness, Sloan (1961) found that absolute thresholds were lowest in the fovea when the eye was adapted to $75 \mathrm{~dB}$ re $10^{-10}$ lambert. She used targets varying from about $28^{\prime}$ to about $50^{\prime \prime}$ visual angle, and all showed a constant increase in the absolute threshold as the target was moved outward from the fovea in both directions along the horizontal meridian.

Table 6. Constants of the Foveal and Peripheral Brightness Functions $\dot{\psi}=k\left(\phi-\phi_{\circ}\right)^{0.33}$. Entries conform with the equal-brightness contours in Fig. 5; $\phi$ and $\phi_{\mathrm{o}}$ are in millilamberts.

\begin{tabular}{ccl} 
Retinal locus & \multicolumn{1}{c}{$k$} & \multicolumn{1}{c}{$\phi_{\mathrm{o}}$} \\
\hline Fovea & 1.00 & 0.005 \\
$5^{\circ}$ & 1.12 & 0.00016 \\
$12^{\circ}$ & 1.21 & 0.00007 \\
$20^{\circ}$ & 1.25 & 0.000025 \\
$35^{\circ}$ & 1.20 & 0.0001 \\
$60^{\circ}$ & 1.19 & 0.0045 \\
\hline
\end{tabular}




\section{References}

Arden, G. B., \& Weale, R. A. Nervous mechanisms and darkadaptation. J. Physiol., 1954, 125, 417-426.

Crozier, W. J., \& Holway, A. H. Theory and measurement of visual mechanisms: I. A visual discriminometer. II. Threshold stimulus intensity and retinal position. J. gen. Physiol., 1939, 22, 341364.

Graham, C. H., Brown, R. H., \& Mote, F. A., Jr. The relation of size of stimulus and intensity in the human eye: I. Intensity thresholds for white light. J. exp. Psychol., 1939, 34, 555-573.

Hecht, S., Haig, C., \& Wald, G. The dark adaptation of retinal fields of different size and location. J. gen. Physiol., 1935, 19, 321-337.

Hecht, S., Schlaer, S., \& Pirenne, M. H. Energy at the threshold of vision. Science, 1941, 93, 585-587.

Onley, J. W. Visual sensitivity. Ann. Rev. Psychol., 1964, 15, 29-56.

Pirenne, M. H. Vision and the eye. London: Chapman and Hall, 1948.

Sloan, L. L. Area and luminance of test object as variables in examination of the visual field by projection perimetry. Vision Res., 1961, 1, 121-138.

Stevens, J. C., \& Stevens, S. S. Brightness function: Effects of adaptation. J. Opt. Soc. Amer., 1963, 53, 375-385.

Stevens, S. S. Calculation of the loudness of complex noise. $J$. Acoust. Soc. Amer., 1956, 28, 807-832.

Stevens, S. S. Cross-modality validation of subjective scales for loudness, vibration, and electric shock. J. exp. Psychol., 1959, 57, 201-209.
Stevens, S. S. The psychophysics of sensory function. In W. A. Rosenblith (Ed.), Sensory communication. Cambridge, Mass.: M. I. T. Press, 1961. Pp. 1-33.

Stiles, W. S., \& Crawford, B. H. The liminal brightness increment for white light for different conditions of the foveal and parafoveal retina. Proc. Roy. Soc., 1934, B116, 55-102.

Stiles, W. S., \& Crawford, B. H. The effect of a glaring light source on extrafoveal vision. Proc. Roy. Soc., 1937, B122, 255-280. Troland, L. T. Psychophysiology. Vol. II. New York: Van Nostrand, 1930.

Wentworth, H. A. A quantitative study of achromatic and chromatic sensitivity from center to periphery of the visual field. Psychol. Monogr., 1930, 40 (Whole No. 183).

Wolf, E., \& Zigler, M. J. Uniocular and binocular scotopic responsiveness of the peripheral retina. J. Opt. Soc. Amer., 1959 , 49, 394-398.

Zigler, M. J., \& Wolf, E. Uniocular and binocular scotopic parafoveal sensitivity. Amer. J. Psychol.. 1958, 71, 186-198.

\section{Notes}

1. Research supported by grants from the National Science Foundation and the National Institutes of Health (Laboratory of Psychophysics report PPR-326).

2. Now at John B. Pierce Foundation Laboratory, and Department of Psychology, Yale University, New Haven, Connecticut.

(Accepted for publication Aug. 15, 1966.) 